\title{
Experimental Study on Healing of Bone Implants Treated by Heating or Freezing
}

\author{
Takesuke Dohtsu, Tsugio Inokuchi, Ryoji Hironaka, Hisazumi Ikeda, Hidenori Ninomiya and Joji Sekine \\ Second Department of Oral and Maxillofacial Surgery, Nagasaki University School of Dentistry, 1-7-1 Sakamoto, Nagasaki 852- \\ 8588, Japan
}

\begin{abstract}
Dohtsu T, Inokuchi T, Hironaka R, Ikeda H, Ninomiya H and Sekine J. Experimental study on healing of bone implants treated by heating or freezing Oral Med Pathol 2001; 6: 43-50, ISSN 1342-0984.
\end{abstract}

\begin{abstract}
The aim of this study was to assess the healing response to the bone implants treated by heating or freezing. Allogenic calvarial implants treated by heating at $65^{\circ} \mathrm{C}$ for 1 hour, 3 freezes of 3 minutes, or boiling for 10 minutes $\left(65^{\circ} \mathrm{C}\right.$, frozen and boiled group respectively) were tested for the potential to fill cranial bone defects of rabbits. Both the $65^{\circ} \mathrm{C}$ and frozen groups similarly showed more than $60 \%$ of new bone formation in terms of the untreated control. Among the three treatment modalities, boiling presented the most remarkable impairment on the reparative capacity of the implants.

The autogenous bone segments reimplanted to their original mandibular sites after treatment by heating at $65^{\circ} \mathrm{C}$ using microwave or by freezing were revealed to similarly incorporate to the host bone in dog experiments. In summary, heat treatment at $65^{\circ} \mathrm{C}$ for 1 hour is comparable with freeze treatment in terms of preservation of the regenerative potential of bone for simultaneous reimplantation.
\end{abstract}

Key words: reimplantation of bone, devitalization of tumor cells, BMPs

Corespondence : Tsugio Inokuchi, Second Department of Oral and Maxillofacial Surgery, Nagasaki University School of Dentistry, 1-7-1 Sakamoto, Nagasaki 852-8588, Japan Phone: +81-95-849-7701, Fax: +81-95-849-7705

\section{Introduction}

Resection of the mandibular arch for cancer involvement results in functional and cosmetic disturbance. Many methods of mandibular reconstruction using bone or alloplastic materials have been described.

Use of resected bone as a graft should ideally provide a solution that satisfies both functional and cosmetic demands, avoiding a possible immune reaction and transmission of diseases.

Extracorporeal treatment such as heating (1-3), freezing (4-6) or irradiation (7) has been employed to devitalize the tumor-infiltrated bone segment prior to reimplantation.

Such a treatment essentially requires complete devitalization of the tumor cells within the bone. However, preservation of the regenerative potential of the implant should also be considered for successful bone healing in the recipient bed compromised by surgery and/ or irradiation.

Osteogenic factors including bone morphogenetic proteins $(\mathrm{BMPs})$ contained in the implant play important roles in bone regeneration (8). Bone induction is produced by the response of the mesenchymal cells in the surrounding tissues to the osteogenic factors transferred from the bone implant. Regarding the effect of thermal exposure on bone implant, Urist and Iwata demonstrated that biological activity of morphogenetic property was completely destroyed by heating at $70^{\circ} \mathrm{C}$ and above for 1 hour (9).

The freeze-treated bone implant is osteoinductive, probably because of preserved osteogenic factors $(5,10)$. On the other hand, moderate heat treatment, i.e., pasteurization, is applicable as an alternative procedure. We proposed heat treatment at $65^{\circ} \mathrm{C}$ for complete devitalization of the tumor cells without destruction of osteoinductive properties, and reported clinical application in the form of immediate reimplantation of the resected mandibular segment (11). However, healing response to this heat-treated implant in the orthotopic site has not been fully investigated.

The aim of this study was to evaluate healing of bone implant treated by heating in comparison with freezing. 


\section{Materials and Methods}

Experiment 1 (Rabbit experiment)

(1) Animals:

Ten male and female Japanese white rabbits, 8 months old, weighing about $2.5 \mathrm{~kg}$, were used. Implant materials were harvested from 2 rabbits and were implanted to 8 rabbits.

Animals were anesthetized with intramuscular injection of ketamine $(35 \mathrm{mg} / \mathrm{kg})$.

One month after surgery, the animals were euthanized with an overdose of pentobarbital sodium.

\section{(2) Preparation of implant materials:}

The allogenic bone implants were prepared from the calvarial bones and the diaphyseal cortical long bones of the euthanized rabbits under aseptic conditions. The bones were defatted in $100 \%$ ethanol and diethyl ether, and decalcified by $0.6 \mathrm{~N} \mathrm{HCl}$, with $1 \mathrm{~g}$ of bone per $200 \mathrm{ml}$ of solution for 1 hour at $25^{\circ} \mathrm{C}$ in an ultrasonic washing machine. After decalcification, the acid was removed by washing in normal saline solution.

(a) Bone discs: The decalcified calvarial bone discs, $8 \mathrm{~mm}$ in diameter, for implantation to the cranial defects, were made using a trephine. These bone discs were subjected to heat, boiling or freeze treatment. The untreated bone discs were used as control.

(b) Bone strips: The decalcified diaphyseal cortical bones were cut in rectangular strips of $11.5 \mathrm{~cm}$. These bone strips were heated in a water bath or a microwave oven and were used for intramuscular implantation.

(3) Heat treatment $\left(65^{\circ} \mathrm{C}\right.$ group):

Bone discs immersed in distilled water were heated at $65^{\circ} \mathrm{C}$ for 60 minutes using a temperature-controlled water bath.

Bone strips were heated at $65^{\circ} \mathrm{C}$ for 60 minutes in a water bath or a microwave oven.

(4) Boiling (boiled group):

The implant materials were boiled in distilled water at $100^{\circ} \mathrm{C}$ for 10 minutes.

(5) Freeze treatment (frozen group):

The implant materials were triple frozen in a liquid nitrogen bath.

Three freezes of 3 minutes each were employed, with intervening 5-minute thaws in room temperature distilled water.

\section{(6) Surgical procedure:}

(a) Implantation to the cranial defect: After exposing the cranial surface by skin incision, four $8 \mathrm{~mm}$ fullthickness (approximately $2 \mathrm{~mm}$ ) trephined skull defects were made in the frontal and parietal bones. (In our preliminary experiment, the $8-\mathrm{mm}$ skull defects healed by the formation of fibrous connective tissue without bony bridging 1 month postoperatively.) Each defect was filled with one of the four calvarial bone disc implant materials : untreated control, heated at $65^{\circ} \mathrm{C}$, boiled or frozen. The skin was then closed with sutures.

(b) Intramuscular implantation of the bone strips: Two groups of the diaphyseal cortical long bone strips, which were heated in a water bath (water bath group), or heated in a microwave oven (microwave group), were inserted into the abdominal wall muscle of the animals. The skin was then closed with sutures. After surgery, tetracycline was injected intramuscularly for labeling.

\section{(7) Tissue preparation for histological analysis:}

The cranial specimens were fixed in $10 \%$ neutral formalin, decalcified in EDTA, trimmed and embedded in paraffin. Sections were cut transversely in the midpart of the implant site and then stained with hematoxylin and eosin. The total area of new bone formed within the defect was quantified through computer-assisted image analysis (NIH Image Version 1.55 program, Macintosh LC II, Apple Computer, Japan) on a photographic tracing of the histologic section. The new bone formation was expressed in ratio to the control, the measured value of which was defined as 1 .

(8) Tetracycline labeling:

Animals were intramuscularly injected with tetracycline hydrochloride $(40 \mathrm{mg} / \mathrm{kg}$ ) once a week for 3 weeks; once immediately after the surgery and 2 times during the following 2 weeks.

(9) Tissue preparation for tetracycline-labeled specimens:

The bone specimens were fixed in $70 \%$ ethanol, dehydrated, and embedded in Technovit 7200 (T7200, Exakt Medical Instruments, Oklahoma City, OK, USA).

Embedded specimens were cut, and polished to 30 $\mathrm{m}$ using the Cutting-Grinding System (Exakt Medical Instruments, Oklahoma City, OK, USA). The slides were viewed under a fluorescence microscope and the fluorescent area was quantified using computer-assisted image analysis on the photographic tracing of the section.

\section{(10) Statistical analysis:}

ANOVA and Fisher's PLSD test were used for statistical analysis. $P$ values less than 0.05 were considered to indicate statistical significance.

\section{Experiment 2 (Dog experiment)}

(1) Animals:

Ten adult mongrel dogs, each weighing about 12 $\mathrm{kg}$, were used. The dogs were young to middle age, as determined by the condition of their dentition, physical status and coat of hair. 


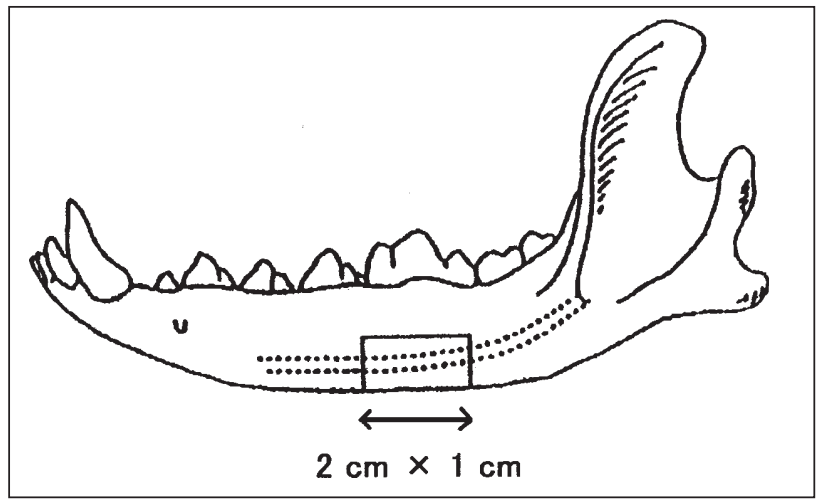

Fig. 1: Surgical sites; The two autogenous osseous implants, 2 $1 \mathrm{~cm}$ in size, were taken from the right and left inferior border of the mandible. The implants were either heated to $65^{\circ} \mathrm{C}$ or frozen, and then reimplanted in their original sites, right and left, respectively (dog experiment).

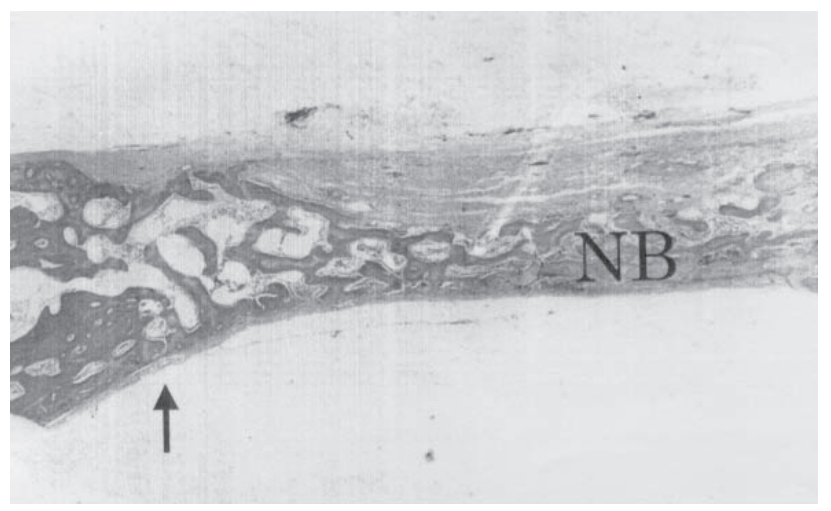

Fig. 3: Bone regeneration of cranial defects implanted with the decalcified bone disc (rabbit experiment), $65^{\circ} \mathrm{C}$ group. Arrow marks original border of defect. NB: new bone, (HE. Original magnification, 2.5)

(2) Preparation of autogenous bone implant:

Two bone segments measuring $21 \mathrm{~cm}$ were taken from both sides of the inferior border of the mandible in the molar regions under general anesthesia with pentobarbital sodium (Fig.1). The inferior alveolar neurovascular bundle was removed from the resected bone segment.

The bone segment from the right side was treated by heating at $65^{\circ} \mathrm{C}$ for 1 hour in a microwave oven $\left(65^{\circ} \mathrm{C}\right.$ group). The segment from the left side was treated by 3 freeze-thaw cycles, being immersed in liquid nitrogen for 3 minutes and allowed to thaw in saline (frozen group).

(3) Reimplantation of autogenous bone implant:

The bone segments treated by heating at $65^{\circ} \mathrm{C}$ or by freezing were reimplanted in their original sites, right and left, respectively, and fixed with transosseous wires.

The animals were euthanized by overdose of pentobarbital sodium at 2, 4, 6, 24 and 30 weeks postoperatively. One of the animals was administered $10 \mathrm{mg} / \mathrm{kg}$ of oxytetracycline hydrochloride intramuscularly to obtain an intravital osseous label at 2 weeks and 1 week before its euthanization.

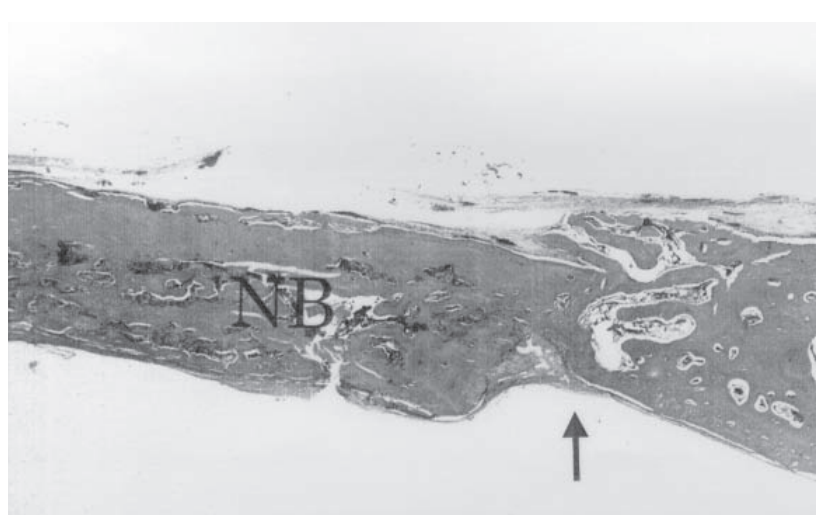

Fig. 2: Bone regeneration of cranial defects implanted with decalcified bone disc (rabbit experiment), untreated control. Arrow marks original border of defect. NB: new bone, (HE. Original magnification, 2.5)

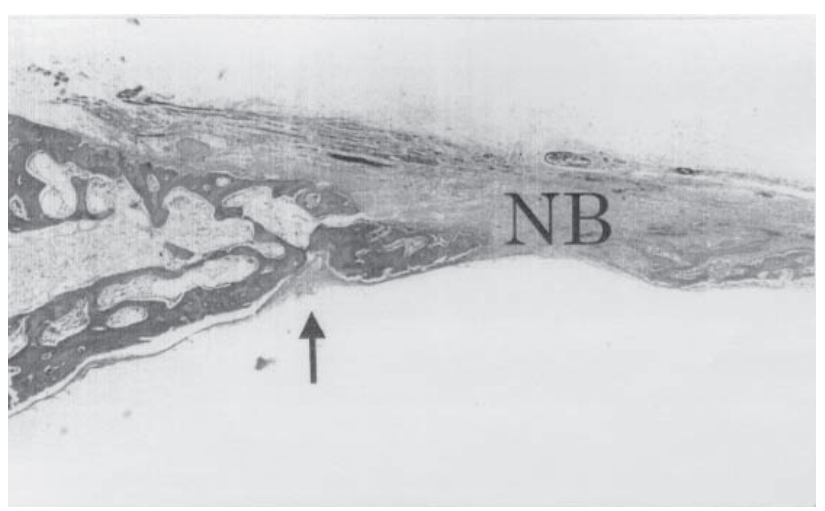

Fig. 4: Bone regeneration of cranial defects implanted with the decalcified bone disc (rabbit experiment), boiled group. Arrow marks original border of defect.NB: new bone, (HE. Original magnification, 2.5)

\section{(4) Tissue preparation:}

The mandibles were processed for histological $\mathrm{HE}$ staining and contact microradiography. Villaneva bone staining was done for the group at 30 weeks after implantation.

\section{Results}

\section{Experiment 1}

(a) Implantation of the bone discs to the cranial defect:

The control group of untreated bone discs showed abundant bone within the defect site (Fig. 2 ). Relatively abundant fibrous tissues were identified in the frozen and the $65^{\circ} \mathrm{C}$ group, although the newly formed bone was less than that presented in the control group (Fig.3).

A fibrous area occurring nearly throughout the cranial defect was observed in the boiled group (Fig. 4). Morphometric evaluation of new bone formation revealed the best results for the control group. The frozen and the $65^{\circ} \mathrm{C}$ groups, which showed no significant difference between them, indicated more than $60 \%$ of the amount of new bone formation presented in the control group. The smallest 
Table 1: Morphometric analysis of defects implanted with heated, frozen or untreated control implant.

\begin{tabular}{c|c|c|c} 
Implant group & $\mathrm{n}$ & New Bone formed & $\mathrm{SD}$ \\
\hline Control & 4 & 1.000 & $0.035 \dagger, \ddagger$ \\
Frozen & 4 & 0.670 & $0.046 *$ \\
$65^{\circ} \mathrm{C}$ & 4 & 0.624 & $0.033 \star \star$ \\
Boiled & 4 & 0.434 & 0.151
\end{tabular}

The total area of bone formed is expressed in ratio to the control whose measured value is defined as 1 .

$\dagger$ : significant compared to frozen implant $(p<0.005)$

\$ : significant compared to $65^{\circ} \mathrm{C}$ and boiled implant $(p<0.001)$

* : significant compared to boiled implant $(p<0.005)$

** : significant compared to boiled implant $(p<0.01)$

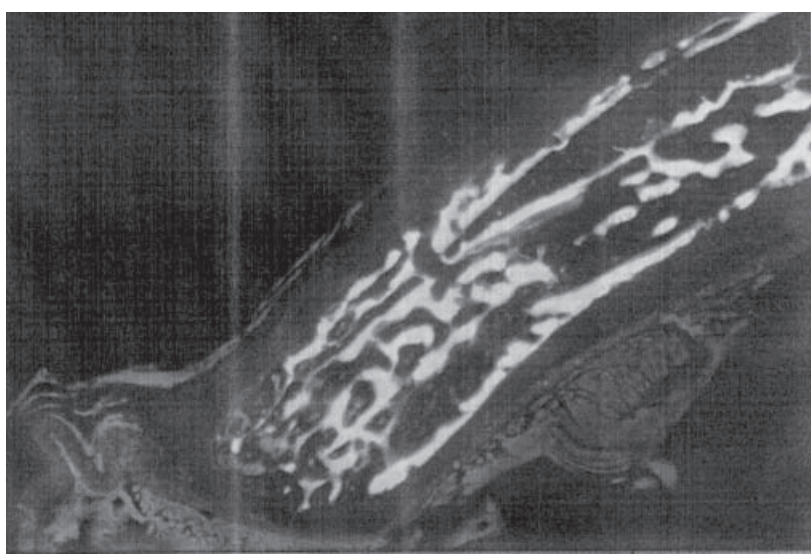

Fig. 5: Bone formation with tetracycline labeling in the intramuscular implantation of decalcified bone strips (rabbit experiment), microwave group. (Original magnification, 2)

amount of new bone formed was observed in the boiled group (Table 1 ).

(b) Implantation of the bone strips to muscle:

There was no significant difference between the implants heated by water bath or microwave in regard to new bone formation (Fig. 5, Table 2).

\section{Experiment 2}

Two weeks after implantation:

Contact microradiography revealed the fine structures of new bone beginning at the medullary and outer surface of the host bone close to the surgical site in both the $65^{\circ} \mathrm{C}$ and the frozen group (Fig. 6).

In microscopic observations, both of the groups showed newly formed immature bone surrounded by osteoblasts in the osteotomy sites. The host bone-implant interface (hereafter referred to as the interface) was filled with new bone extending from the host bone marrow. Os-
Table2: Morphometric analysis of new bone formation in intramuscular implantation of the decalcified allogenic bone.

\begin{tabular}{c|c|c|c} 
Group & $\mathrm{n}$ & $\begin{array}{c}\text { New Bone } \\
\text { mean }\end{array}$ & S.D. \\
\hline Microwave & 12 & 1.106 & 0.369 \\
\hline Water Bath & 12 & 1.000 & 0.308
\end{tabular}

The new bone formed is expressed in ratio to the water bath group, whose measured value is defined as 1 .

There was no significant difference between the water bath group and microwave group.

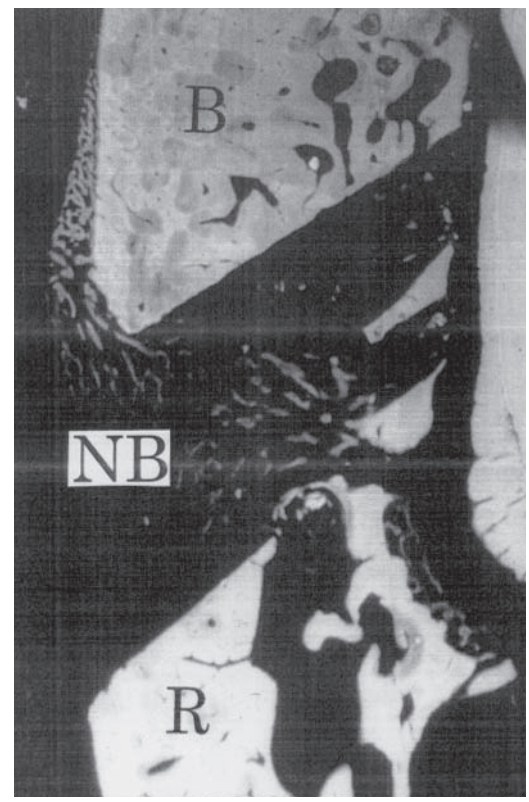

Fig. 6: Reimplanted mandibular bone segment 2 weeks after surgery (dog experiment), $65^{\circ} \mathrm{C}$ group. The fine structures of new bone are presented in the surgical site.

B: host bone, R: reimplanted bone, NB: new bone (contact microradiography)

teoclastic activity was also noted in the interior of the implant. The trabeculae, hematopoietic marrow and vascular spaces were partly presented within the new bone adjacent to the host bone, while more immature features with osteoid tissues and smaller marrow spaces were shown in the part distal to the host bone (Fig. 7). The medullary space of the implant was filled with the fibrous tissues containing abundant small vessels. The outer surface of the implant showed mottling, with howship's lacunae containing osteoclasts.

Four weeks after implantation:

Periosteal and endosteal bone proliferation from the host bone was evident. Osteoid fusion to the implant and bridging between the host bone and the implant were demonstrated (Fig. 8).

Six weeks after implantation:

Both groups showed similar findings in contact microradiography. The bone proliferation from the host 


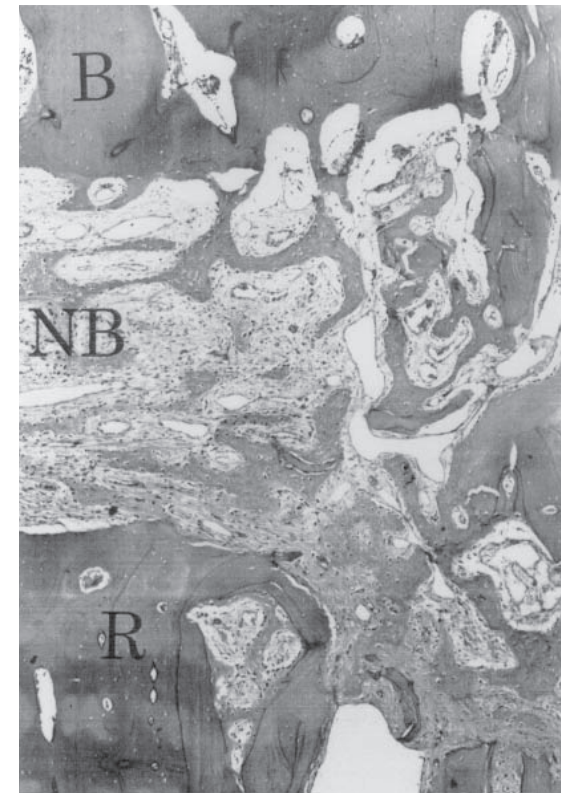

Fig. 7 : The interface showing osteoblastic and osteoclastic activity 2 weeks after surgery (dog experiment), frozen group. B: host bone, R: reimplanted bone, NB: new bone (HE. Original magnification, 16)

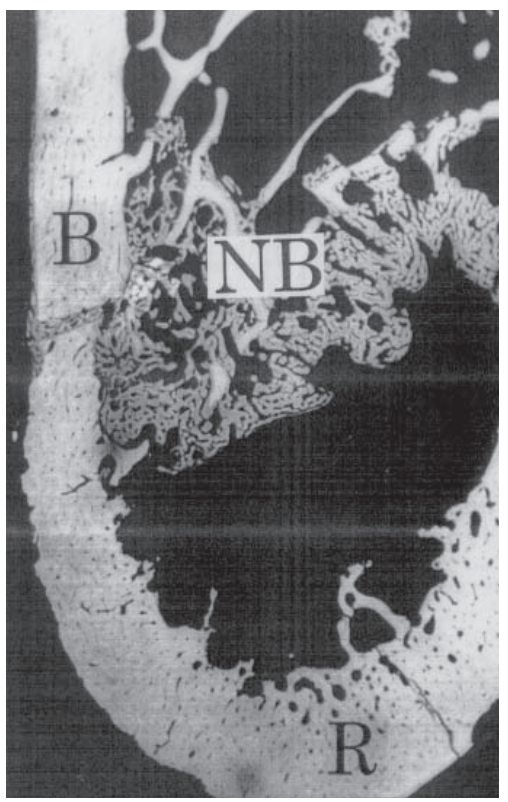

Fig. 9 : Union between the reimplanted mandibular bone segment and the host bone 6 weeks after surgery (dog experiment), $65^{\circ} \mathrm{C}$ group. B: host bone, R: reimplanted bone, NB: new bone (contact microradiography)

bone was more advanced in the medullary space, the interface and on the outer surface of the implant bone. Union between the host bone and the implant was apparent. Compared with the findings at 2 weeks after implantation, the new bone demonstrated increased radiopacity and trabecular width. The implants showed osteoporotic features with enlarged haversian canals. On the periosteal surface of the host bone, lamellar bone formation was evident (Fig. 9). Microscopic evaluation revealed that the implant was well incorporated into the host bone at the

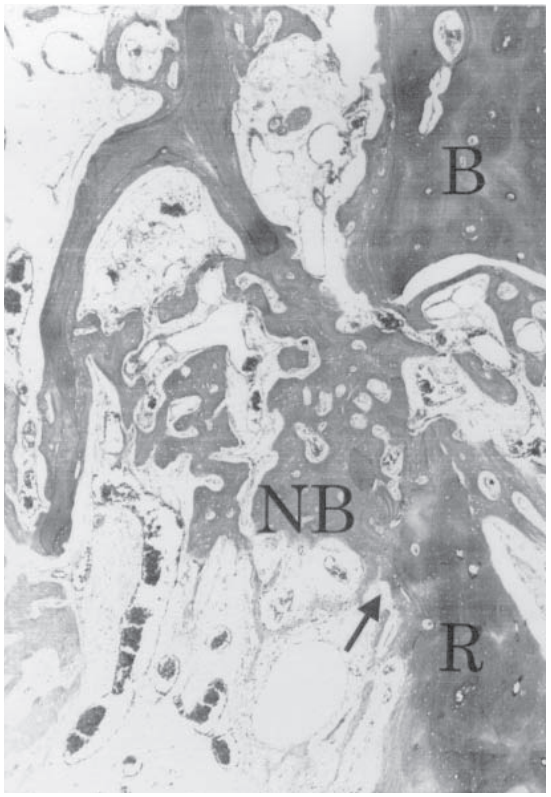

Fig. 8 : Reimplanted mandibular bone segment 4 weeks after surgery (dog experiment), $65^{\circ} \mathrm{C}$ group.

Note fusion of the new bone to the implant in the medullary site adjacent to the interface (arrow). B: host bone, R: reimplanted bone, NB: new bone (HE. Original magnification, 10)

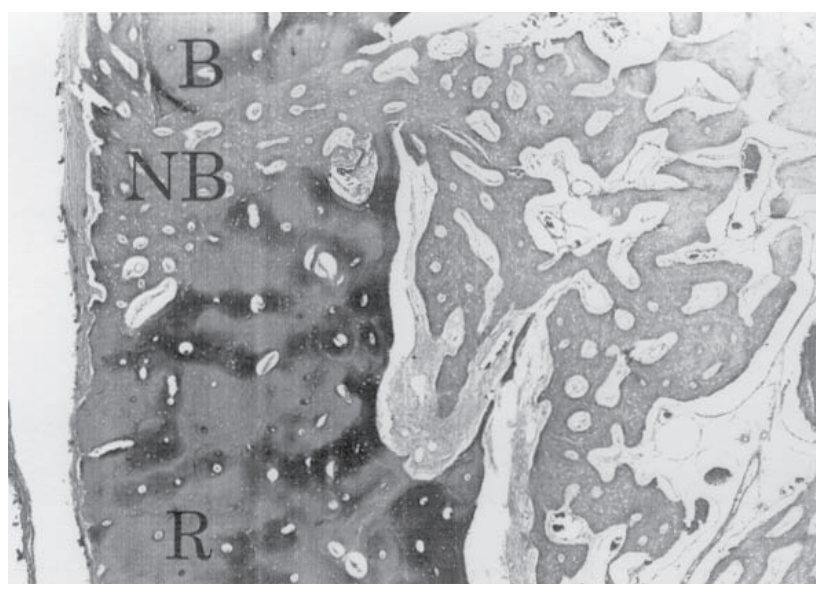

Fig. 10:Reimplanted mandibular bone segment 6 weeks after surgery(dog experiment), $65^{\circ} \mathrm{C}$ group. The edge of the implant is replaced by new bone. B: host bone, $\mathrm{R}$ : reimplanted bone, NB: new bone (HE. Original magnification, 10)

osteotomy site, where the implant was replaced by the newly formed bone for both groups. The new bone at the interface demonstrated progressed maturation of the trabeculae and marrow spaces (Fig. 10). On the other hand, the immature bone was still noted to be proliferating in the medullary space distal to the host bone (Fig. 11).

Twenty four weeks after implantation:

Smooth continuity from the host bone to the implant resulting from periosteal bone proliferation was demonstrated in contact microphotography for both groups. The inner side of the implants, for both groups, 


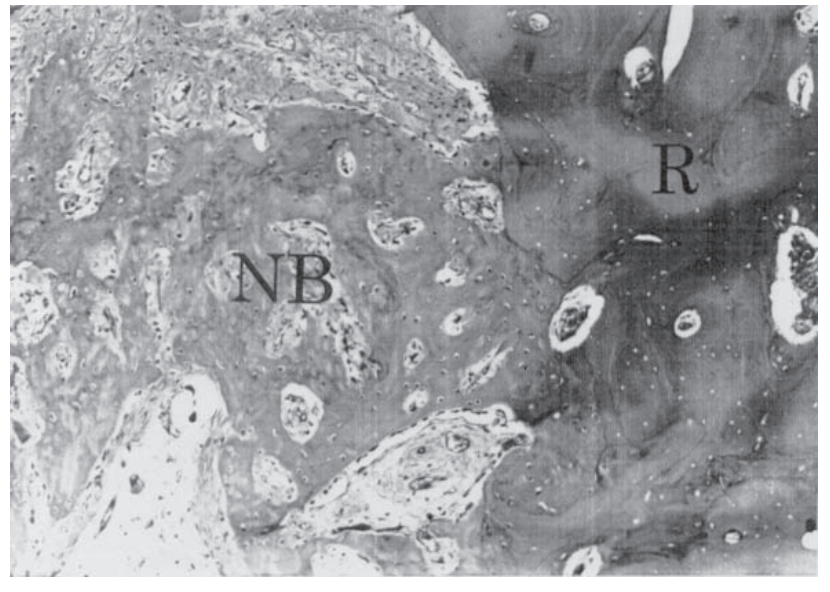

Fig. 11: Immature bone occupying the medullary space of the implant distant from the host bone in 6-week specimen (dog experiment), frozen group. R: reimplanted bone, NB: new bone (HE. Original magnification, 25)

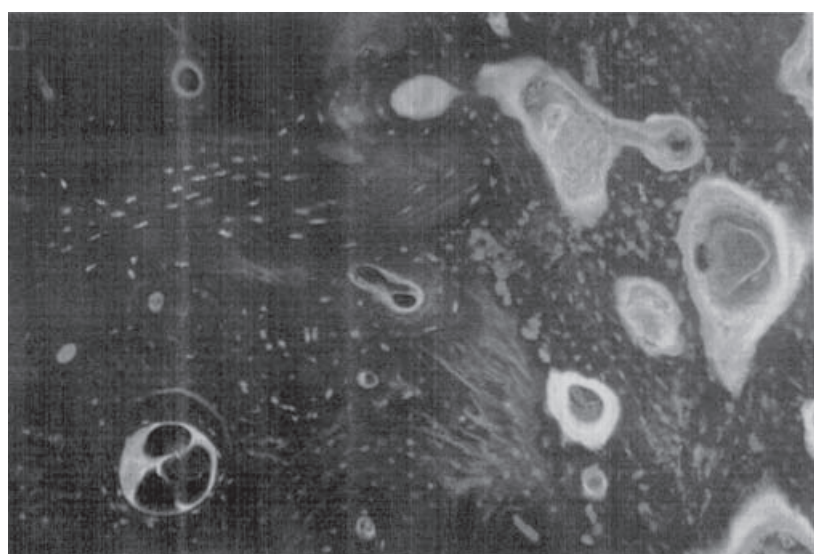

was replaced by new haversian osteons, and remarkable formation of the trabecular bone occurred in the medullary space (Fig. 12).

Thirty weeks after implantation:

In both groups, Villaneva bone stain revealed the area labeled with tetracycline and osteoid stained red in the interface and the medullary space. Tetracycline-labeled parts were also observed in the haversian canals of the implant (Fig. 13).

\section{Discussion}

Implantation to the cranial defect

The present study was designed to investigate the effect of devitalization treatment by heating or freezing on the healing of decalcified allogenic bone implants, the osteoinductive property of which was discovered by Urist $(12,13)$. The experiment involving implantation to the cranial defect indicates that new bone formation is affected by all the treatments including boiling at $100^{\circ} \mathrm{C}$ for 10 minutes, heating at $65^{\circ} \mathrm{C}$ for 1 hour and freezing, as compared with the untreated control. Boiling showed the most remarkable impairment of implant healing among these 4 groups. More than $60 \%$ of new bone for-

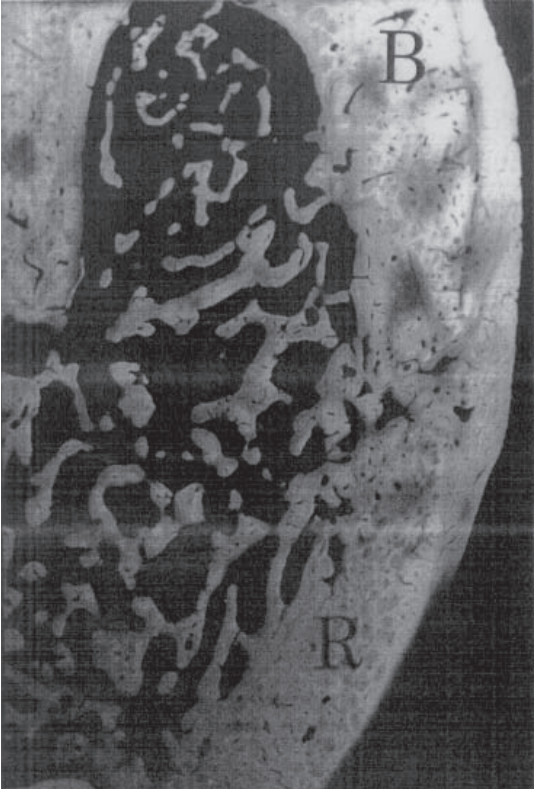

Fig. 12: Reimplanted mandibular bone segment 24 weeks after surgery (dog experiment), $65^{\circ} \mathrm{C}$ group. Periosteal bone proliferation showing smooth continuity from the host bone to the implant (contact microradiography). Formation of the travecular bone in the medullary space of the implant is demonstrated. B: host bone, R: reimplanted bone, NB: new bone (contact microradiography)

Fig. 13: Reimplanted mandibular bone segment 30 weeks after surgery (dog experiment), $65^{\circ} \mathrm{C}$ group. Tetracycline labeling is shown in the haversian canals of the implant (Villaneva bone stain, 25).

mation in terms of the control was presented in response to the implants of the $65^{\circ} \mathrm{C}$ group and the frozen group. These observations suggest that the osteogenetic property of the implant is relatively preserved in the devitalization treatment by moderate heating, pasteurization or freezing.

Regarding the thermal effect on the bone implant, we reported in our previous study that treatment at $65^{\circ} \mathrm{C}$ for 30-120 minutes preserves the osteoinductive property and completely kills tumor cells (11). Ito, based on the expression of mRNA of alkaline phosphatase and collagen I and II, reported that heating at $60^{\circ} \mathrm{C}$ for less than 10 hours and even at $70^{\circ} \mathrm{C}$ for less than one hour preserve osteoinductive activity of bone implant (14). Heating by high temperature, boiling or autoclaving completely destroys the inductive repair capacity (9, 11-13). Additionally, high temperature treatment leads to coagulation of the haversian canals and denaturation of bone collagen, coagulated tissues filling the haversian canals impair revascularization $(15,16)$, and denatured bone matrix impeding the normal biologic repair process $(16,17)$.

The present study showed that heating at $65^{\circ} \mathrm{C}$, as a treatment for bone implant, is almost the same as freez- 
ing in terms of the osteogenic response of the host. Although the amount of newly formed bone appears to be fairly sufficient, this moderate heat treatment is advantageous in comparison with boiling as a devitalization technique for the bone to be reimplanted. In this experiment, the bone discs were implanted into the orthotopic site, i.e., the cranial bone defect. It is assumed, therefore, that the osteogenetic regeneration observed was through both osteoinductive and osteoconductive activities in the implantation site.

Heat treatment using microwave

In our previous study, we reported a heat treatment technique using a microwave oven, which is advantageous in the preparation of bone implant in comparison to heating by a water bath (11). It is known that microwave irradiation stabilizes tissue proteins and induces cross-linking of collagen $(20,21)$. The increased strength of the protein network may resist biological degradation or remodeling of implanted tissue. However, the effect of heating by microwave on the osteogenetic property of the bone implant remains undefined.

In the experiment involving intramuscular implantation of the bone strips, we compared 2 heating methods (heating by water bath or microwave) with respect to the effect on the bone induction potential of the implant.No significant difference was observed between these 2 heating methods in terms their effect on bone induction of the implant. This result suggests that osteoinductive factors including the BMPs are preserved in the bone implant heated by microwave as well as in that heated by a water bath.

\section{Reimplantation of bone after devitalization treatment}

Reuse of the resected tumorous bone after devitalization of tumor cells is an attractive alternative modality in bone reconstruction. Autoclaving of bone is assumed to cause extensive denaturation of proteins, including the bone inductive proteins $(9,22)$. On the other hand, freeze-treated bone has been reported to be comparable to fresh bone graft in terms of osteogenic potential $(23,24)$. This study was to investigate the fate of bone implants treated by moderate temperature (pasteurization) in comparison with freeze-treated bone implants.

In both the frozen and heated groups, periosteal and endosteal new bone formation commenced at the host bone side, and proceeded toward the implant.Early fusion between the implant and the host bone occurred 4 weeks after surgery, and incorporation of the implant to the host bone was clearly demonstrated at the interface at 6 weeks. In the medullary space, on the other hand, new bone formation advanced to the implant side. These findings indicate conductive healing of the bone.

Neither the heated nor the frozen bone implants were fully substituted even 7 months after implantation, and Villaneva staining revealed the implants to be still in the remodeling process. The new bone formation presented around the haversian canals may suggest that substitution of bone implant also proceeds through the haversian canals.

In surgery of benign tumorous bone such as that of ameloblastoma, the surrounding periosteum can usually be preserved, and its excision limited to the part invaded by the tumor.

Consequently, this surgical site with the periosteum preserved is advantageous to healing of the implanted bone. Incorporation by periosteal osteogenesis observed in this study indicates such a clinical bone repair.

In contrast to surgery for benign tumorous bone, cancer infiltrated bone must be resected with the surrounding soft tissues, including the periosteum as part of tumor extirpation. This surgical intervention results in providing a nutritionally poor environment as the recipient bed for reconstructive bone grafting, and radiotherapy further decreases vascularity. The present study indicates that bone segment devitalized by moderate heat treatment (pasteurization) or freezing can be used as a cortical stent which may provide osteogenetic activity in the compromised recipient bed. In addition, provision of supplementary bone marrow or osteoinductive substance to the bone implant may be advantageous to improve osseous repair (6, 8, 25-29).

In summary, heat treatment at $65^{\circ} \mathrm{C}$ for 1 hour is comparable with freeze treatment in terms of preservation of the regenerative potential of bone for simultaneous reimplantation.

\section{References}

1. Alvarado H A, Casanova-Diaz AS, Capella A, et al. Mandibular reconstruction in patient with oral carcinoma using autogenous mandibular implants.Oral Surg 1970; 30: 11-20.

2. Ewers R and Wangerin K. The autoclaved autogenous reimplant, an immediately replaced, mineral frame. J Maxillofac Surg 1986; 14: 138-142.

3. Lauritzen C, Alberius P, Santanelli F, et al. Repositioning of craniofacial tumorous bone after autoclaving. Scand J Plast Reconstr Hand Surg 1991; 25: 161-165

4. Dougherty TP, Rafetto LK, Edwards RJ, et al. Reimplantation of freeze-treated bone in immediate reconstruction of the mandible. Am J Surg 1982; 144: 463-465.

5. Leipzig B and Cummings CW. The current status of mandibular reconstruction using autogenous frozen mandibular grafts. Head Neck Surg 1984; 6: 992-997.

6. Bradley PF. The cryosurgery of bone in the maxillofacial region. In:Bradley PF ed. Cryosurgery of the Maxillofacial Region, vol. II. CRC Press, Florida.1986; 55-91.

7. Hamaker RC, Singer MI, Shockley WW, et al. Irradiated mandibular autografts. Cancer 1983; 52: 1017-1021.

8. Linkhart TA, Mohan S and Baylink DJ. Growth factors for bone growth and repair: IGF, TGF- $\beta$ and BMP. Bone 1996; 19 (Suppl):1S-12S. 
9. Urist MR and Iwata H. Preservation and biodegradation of the morphogenetic property of bone matrix. J Theor Biol 1973; 38: 155-167.

10. Marciani RD, Giansanti JS and Massey GB. Reimplantation of freeze-treated and saline-treated mandibular bone. J Oral Surgery 1976; 34: 314-319.

11. Inokuchi T, Ninomiya H, Hironaka R, et al. Studies on heat treatment for immediate reimplantation of resected bone. $J$ Cranio Max Fac Surg 1991; 19: 31-39.

12. Urist MR. Bone formation by autoinduction. Science 1965; 150: 893-899.

13. Urist MR and Strates B. Bone morphogenetic protein. J Dent Res 1971; 6: 1392-1406.

14. Ito T, Sakano S, Sato K, et al. Sensitivity of osteoinductive activity of demineralized and defatted rat femur to temperature and duration of heating. Clin Orthop Relat Res 1995; 316: $267-275$.

15. Deleu J. Vascularization of bone grafts in the anterior chamber of the eye. J Bone Joint Surg 1965; 47B: 319-329.

16. Burchardt H. The biology of bone graft repair. Clin Orthop 1983; 174: 28-42.

17. Burwell, RG. Studies in the transplantation of bone VIII. Treated composite homo-autografts of cancellous bone. $J$ Bone Joint Surg 1966; 48B: 532-566.

18. Glowaki J, Altobelli D, Mulliken JB. The fate of mineralized and demineralized osseous implants in cranial drfects. Calcif Tissue Int 1981; 33: 71-76.

19. Scott CK and Hightower JA. The matrix of endochondral bone differs from the matrix of intramembranous bone. Calcif Tissue Int 1991; 49: 349-354.

20. Hopwood D, Coghill G, Ramsay J, et al. Microwave fixation: Its potential for routine technique, histochemistry, and elec- tron microscopy. Histochem J 1984; 16: 1171-1191.

21. Visser CE, Voute AB, Oosting J, et al. Microwave irradiation and cross-linking of collagen. Biomatrials. 1992; 13: 34-37.

22. Urist MR and Strates BS. Bone morphogenetic protein. $J$ Dent Res 1971; 50 (Suppl): 1392-1406.

23. Marciani RD and Bowden CM. Reimplantation of freezetreated mandibular bone. J Oral Surgery 1975; 33: 261-267.

24. Marciani RD, Giansani JS and Massey GB. Reimplantation of freeze-treated and saline-treated mandiblar bone. J Oral Surgery 1976; 34: 314-319.

25. Chesmel KD, Branger J, Wertheim H, et al. Healing response to various forms of human demineralized bone matrix in athymic rat cranial defects. J Oral Maxillofac Surg 1998; 56: 854-863.

26. Rabie A B M, Wong R W K and Hagg U. Composite autogenous bone and demineralized bone matrices used to repair defects in the parietal bone of rabbits. British $\mathrm{J}$ Oral Maxillofac Surg 2000; 38: 565-570.

27. Yamaguchi A, Komori T and Suda T. Regulation of osteoblast differentiation mediated by bone morphogenetic proteins, hedgehogs, and Cbfa1. Endocr Rev 2000; 21: 393-411.

28. Zellin G, Alberius P and Linde A. Autoclaved bone for craniofacial reconstruction: Effects of supplementation with bone marrow or recombinant human fibroblast growth factor-2. Plast Reconstr Surg 1998;102: 792-800.

29. Si X, Jin Y and Yang L. Induction of new bone by ceramic bovine bone with recombinant human bone morphogenetic protein 2 and transforming growth factor $\beta$. Int J Oral Maxillofac Surg 1998; 27: 310-314.

(Accepted for publication May 14, 2001) 